\title{
Influence of Experimental Conditions on the Incorporation of Water Droplets in Polystyrene
}

\author{
Carlos H. Neves ${ }^{\dagger}$, Ricardo A. F. Machado, Ariovaldo Bolzan \\ Departamento de Engenharia Química, UFSC
}

\author{
Odinei H. Gonçalves \\ Universidade Federal Tecnológica do Paraná
}

\begin{abstract}
The substitution of volatile hydrocarbons by the use of water as blowing agent in the synthesis of expandable polystyrene particles has been studied in the last decades. In this work, an investigation was made of the influence of experimental parameters on the incorporation of water in the synthesis of water-expandable polystyrene. The synthesis procedure was based on a two-step polymerization, namely a water dispersion stage and a suspension polymerization stage. The use of a surfactant with a low HLB value resulted in a more stable water-in-oil system and smaller water droplets. The type of impeller used during the dispersion of the water in the monomer phase also influenced the formation of water droplets. In addition, the diameter of the droplets was strongly influenced by the duration of the water dispersion phase. Results suggested that these parameters can be used to control the characteristics of the water droplets formed inside the polystyrene particles.
\end{abstract}

Keywords: Expandable polystyrene, blowing agent, WEPS.

\section{Introduction}

A considerable effort has been dedicated in the last decades to produce expandable polystyrene using renewable, non-toxic blowing agents in order to minimize or even eliminate the use of blowing agents based on volatile hydrocarbons ${ }^{[1,2,4]}$.

Expanded polystyrene is used worldwide in food packaging. The mayor problem is the presence of volatile hydrocarbons that remains in the foam after molding because the residual hydrocarbon can migrate to the food compromising its quality. Although the use of water would probably require more energy it could be a solution to the safety concerns on food package.

The use of water as blowing agent for expandable polystyrene appears as a promising alternative to substitute the conventional blowing agents. However, water incorporation is a challenging task since water is too polar to be able to diffuse into the polystyrene matrix. Some works reported different methods to incorporate water in polystyrene particles. Wittenberg (1992) ${ }^{[1]}$ described a methodology to incorporate water from the continuous suspension medium as small droplets inside the polystyrene particles using from $0.05 \mathrm{wt} \%$ to $0.20 \mathrm{wt} \%$ of polar polymers as poly(acrylic acid). However, satisfactory expandability was only observed when at least $5.0 \mathrm{wt} \%$ of volatile hydrocarbons were used. In a similar way, Berghmans (2000) ${ }^{[4]}$ used polar polymers to incorporate water in the polymer matrix but in this case only $0.5 \mathrm{wt} \%$ of volatile hydrocarbons was needed. In both cases, water concentration was high near the surface of the particles and decreased near their center resulting in a non-uniform expanded structure.

Crevecoeur et al. (1999a) ${ }^{[2]}$ proposed a process using only water as blowing agent, totally avoiding the use of volatile hydrocarbons. The process was divided in two stages: the first one was a water dispersion stage in which water was added in the reactor with the monomer, the initiator and a suitable surfactant under strong stirring at the polymerization temperature. The second one was a suspension stage, when the continuous suspension medium (water and a suspension stabilizer) was added and the system was allowed to react for more 4 hours. Up to $11 \mathrm{wt} \%$ of water was homogeneously incorporated in the particles by this method. In another work ${ }^{[3]}$, the authors demonstrated that the internal structure of the water expanded polystyrene was very similar to that of the pentane expanded one.

One can observe in the works described above that the water incorporation step is of mayor importance because it determines the final morphology of the incorporated water droplets. In turn, the water droplets morphology determines the expansion behavior of the material as well the cell formation since each cell is originated from one single droplet. Furthermore, water must be homogeneously incorporated along the polystyrene particle to assure that a homogeneous expanded structure will be obtained. It can also be noted that a reduced number of works reported the production of expandable polystyrene using only water as blowing agent. However, the influence of several

\footnotetext{
$\dagger$ in memoriam
}

Autor para correspondência: Odinei H. Gonçalves, Universidade Federal Tecnológica do Paraná, CEP: 87301-006, Campo Mourão, PR, Brasil. 
experimental parameters on water incorporation remains to be investigated. For instance, the type of surfactant used in the water dispersion stage may influence the final water droplets distribution. Another important factor in the water dispersion stage is the turbulence level that will affect the breakage and coalescence of the water droplets and hence their size.

In this work the influence of experimental parameters on the incorporation of water in the synthesis of water expandable polystyrene was studied. The methodology used for the synthesis was based on that proposed by Crevecoeur et al. $(1999 \mathrm{a}, \mathrm{b})^{[1,2]}$ because it uses only water as blowing agent and the foam structure formed is similar to that of commercial expanded polystyrene. The parameters evaluated were the type of impeller, the surfactant used to promote the water incorporation and the duration of the water dispersion stage.

\section{Materials and Methods}

\section{Synthesis}

Styrene (Innova, 12 ppm p-tert-butylcatechol) was used as monomer. Benzoyl peroxide (BPO, Sigma-Aldrich, 98\% minimum purity) was used as initiator; poly(vinyl pyrrolidone)

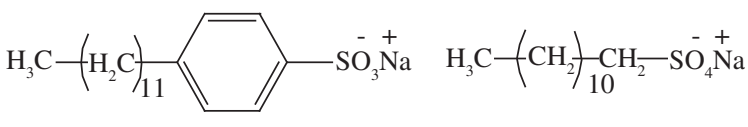

(a)

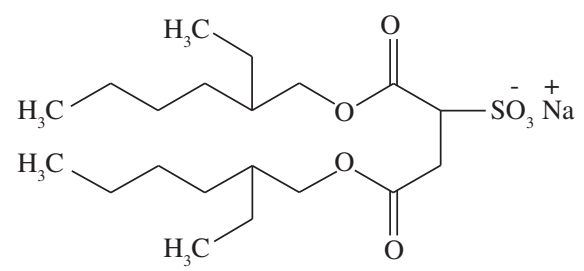

(c)

Figure 1. Chemical structures of a) DBSS; b) DSS; and c) AOT.

Table 1. Formulation used in all experiments.

\begin{tabular}{|c|c|}
\hline Reactant & Concentration (\%) \\
\hline $\mathrm{BPO}^{\dagger}$ & 0.390 \\
\hline $\mathrm{PVP} *$ & 1.000 \\
\hline Surfactant ${ }^{\dagger}$ & 0.100 \\
\hline Water - blowing agent ${ }^{\dagger}$ & 22.000 \\
\hline $\mathrm{NaCl} * *$ & 0.900 \\
\hline
\end{tabular}

Relative to styrene mass; *relative to water mass (suspension medium); **relative to water mass (blowing agent).
(PVP, 360,000 g. mol $^{-1}$ ) was used as suspension stabilizer. Distilled water containing $0.9 \mathrm{wt} \%$ sodium chloride (Vetec, $98 \%$ purity) was used as blowing agent. The surfactants tested were dodecyl benzene sodium sulfonate (DBSS, Vetec, $98 \%$ purity), dodecyl sodium sulfate (DSS, Vetec, 98\% purity) and sodium bis(2-ethylhexyl)sulfosuccinate (AOT, Sigma-Aldrich, 99\% purity). Their chemical structures are presented in Figure 1. Distilled water was used as suspension medium. All reactants were used as received.

Experiments were carried out in a one liter jacketed glass reactor. Reaction temperature was monitored by a $\mathrm{J}$ type thermocouple and controlled by a thermostatic bath. Stirring rate was monitored by an optical sensor and maintained by a frequency controller. Gaseous nitrogen was injected in the reactor to remove the atmospheric oxygen. The formulation used in all experiments is summarized in Table 1, with a styrene volumetric fraction in the suspension of 0.3 . Table 2 presents the experimental conditions used in each experiment.

The polymerization technique adopted in this work is similar to that proposed by Crevecoeur et al. (1999a) ${ }^{[2]}$. The procedure is divided in two stages: water dispersion phase and suspension phase. In the water dispersion phase, water (blowing agent), sodium chloride, surfactant and initiator were mixed in the monomer at $800 \mathrm{rpm}$, resulting in tiny water droplets distributed in the monomer phase. The system was allowed to react at $90{ }^{\circ} \mathrm{C}$ for 135 or 150 minutes (depending of the experiment). In the suspension phase, the continuous medium of the suspension (water and PVP) was added to the reactor. The system was maintained at $90{ }^{\circ} \mathrm{C}$ for more 6 hours.

\section{Characterization}

The amount of water incorporated in the polystyrene particles was determined by thermogravimetric analysis (TGA, Shimadzu TGA-50) under nitrogen supplied at $50 \mathrm{~mL} / \mathrm{min}$. The heating program was as follows: heating rate of $20{ }^{\circ} \mathrm{C} / \mathrm{min}$ until the temperature reached $120{ }^{\circ} \mathrm{C}$; one hour at $120{ }^{\circ} \mathrm{C}$; heating rate of $20{ }^{\circ} \mathrm{C} / \mathrm{min}$ until the temperature reached $180{ }^{\circ} \mathrm{C}$, being maintained for one hour. Scanning Electron Microscopy (SEM, Philips 30) was used to qualitatively evaluate the water incorporation. Particles were immersed in liquid nitrogen and then cut in order to expose the internal microstructure before imaging. Droplets size distribution was determined by measuring about 200 droplets for each experiment.

Table 2. Experimental conditions used in the experiments.

\begin{tabular}{cccccc}
\hline Reaction & Stirring rate $(\mathbf{r p m})$ & Temperature $\left({ }^{\circ} \mathbf{C}\right)$ & Surfactant & Stirrer & Water dispersion time $(\mathbf{m i n u t e s})$ \\
\hline 1 & 800 & 90 & DBSS & Propeller & 150 \\
2 & 800 & 90 & AOT & Propeller & 150 \\
3 & 800 & 90 & DSS & Propeller & 150 \\
4 & 800 & 90 & DSS & Turbine & 150 \\
5 & 800 & 90 & AOT & Propeller & 135 \\
\hline
\end{tabular}




\section{Results and Discussion}

Thermogravimetric analysis indicated a concentration of about $8 \mathrm{wt} \%$ of water in the polystyrene particles in all experiments. This indicates that a fraction of the water-blowing agent added in the system was not effectively incorporated in the polystyrene matrix. This occurred probably due to the loss of blowing agent from the monomer droplets to the suspension continuous phase during the breakage of the monomer droplets in the suspension polymerization stage.

In addition, a homogeneous distribution of the water droplets in the polymer matrix was obtained regardless of the surfactant type, the impeller type or the water dispersion time used. As discussed in other works ${ }^{[3]}$ it is of key importance because the morphology of the expanded polystyrene is largely determined by the distribution of the water droplets in the polymer particle. Particles with non homogeneous droplets distribution usually lead to non uniform expanded foams.

\section{Influence of the impeller type}

Figure 2 presents the internal morphology of the particles obtained using two different impeller types for the dispersion

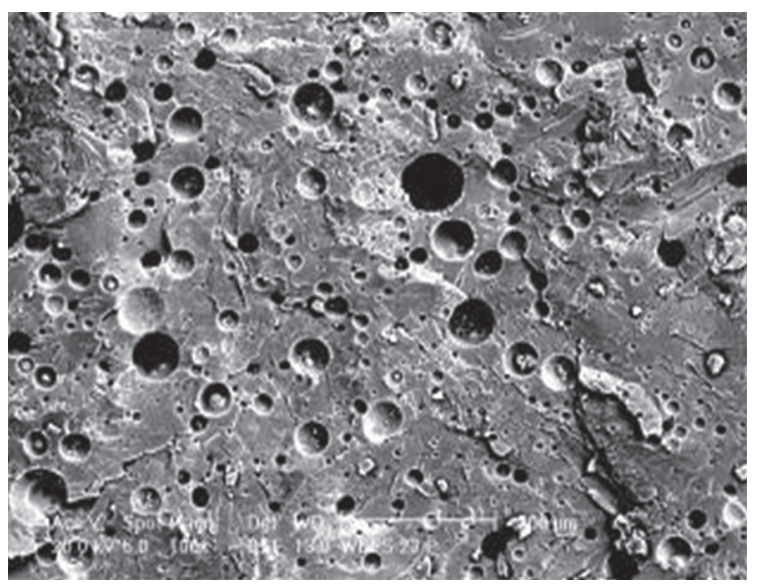

(a)

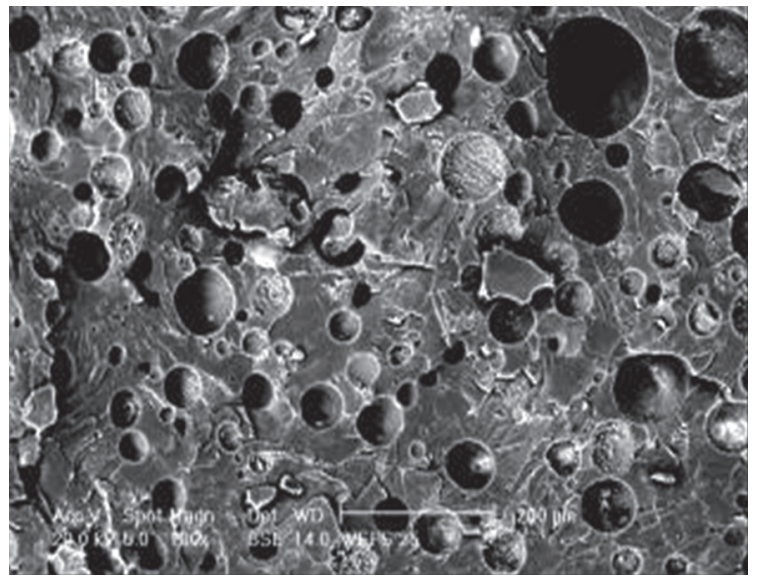

(b)

Figure 2. Micrographs of the material produced using different types of impellers, ( $90^{\circ} \mathrm{C}, 800 \mathrm{rpm}$, DSS, water dispersion time of 150 minutes). a) Turbine impeller $(100 x)$; and b) three-bladed propeller stirrer $(100 \times)$.

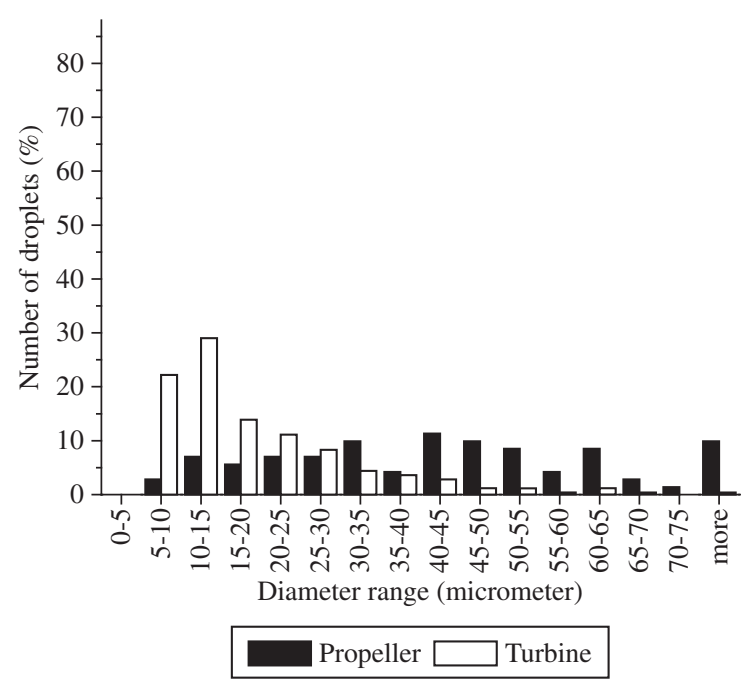

Figure 3. Droplets size distribution obtained using different stirrers $\left(90^{\circ} \mathrm{C}\right.$, $800 \mathrm{rpm}$, DSS, dispersion time of 150 minutes).

of the water in the organic phase, namely three-bladed propeller stirrer and turbine impeller. Figure 3 presents the respective droplets size distribution.

One can observe that the droplets were homogeneously distributed along the particle matrix, since the Scanning Electron Microscopy images were obtained from polymer particles with about 1,000 micrometers diameter.

Average droplet diameter was smaller for the turbine type impeller than for the propeller impeller because. As discussed by Nogueira et al. (2006) ${ }^{[6]}$, the type of impeller affects the mechanisms of breakage and coalescence of the dispersed phase in heterogeneous systems. The geometry of the turbine impeller favored the breakage of water droplets during the water dispersion phase due to the higher shear forces promoted by the perpendicular flux through the turbine impeller resulting in smaller droplets.

\section{Influence of water dispersion time}

Figure 4 presents the internal morphology of the particles obtained using two different times of the dispersion of water in the organic phase (images were taken at different magnifications due to the difference in the droplets diameter). Figure 5 presents the respective droplets size distribution.

The small increase in the duration of the water dispersion step from 135 to 150 minutes led to a decrease in the water droplets diameter. This influence can be explained by the fact that during the water dispersion stage the system is composed by water droplets in the monomer-polymer phase formed by the agitation. At the end of the water dispersion stage, the continuous medium of the suspension (water and the suspension stabilizer) was added to the reactor and the system was then formed by the continuous suspension phase containing monomer-polymer droplets, which in turn contain micro-sized water droplets. The higher the water dispersion time the higher will be the monomer conversion and, consequently, the polymer/monomer ratio and viscosity at 
the beginning of the suspension stage. During the suspension polymerization stage, the levels of turbulence inside the monomer-polymer droplets are low and water droplets are prone to coalesce with each other. The water dispersion time influenced the water droplets size because the water droplets are less likely to coalesce when the viscosity of the monomer-polymer phase is high.

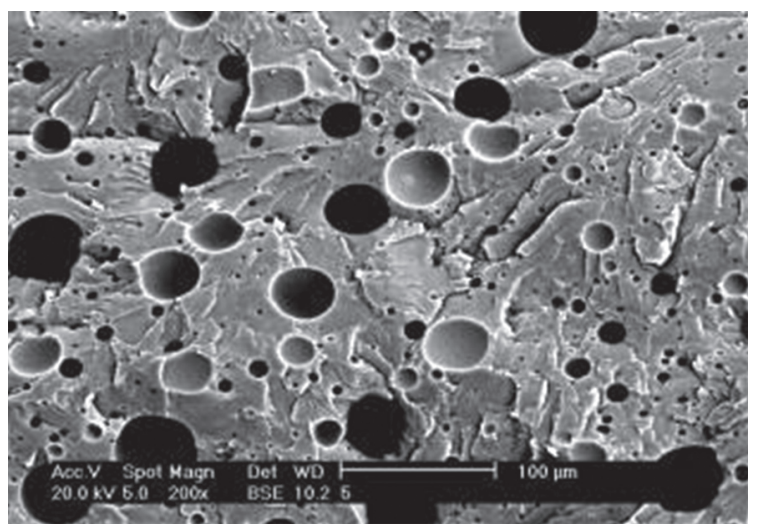

(a)

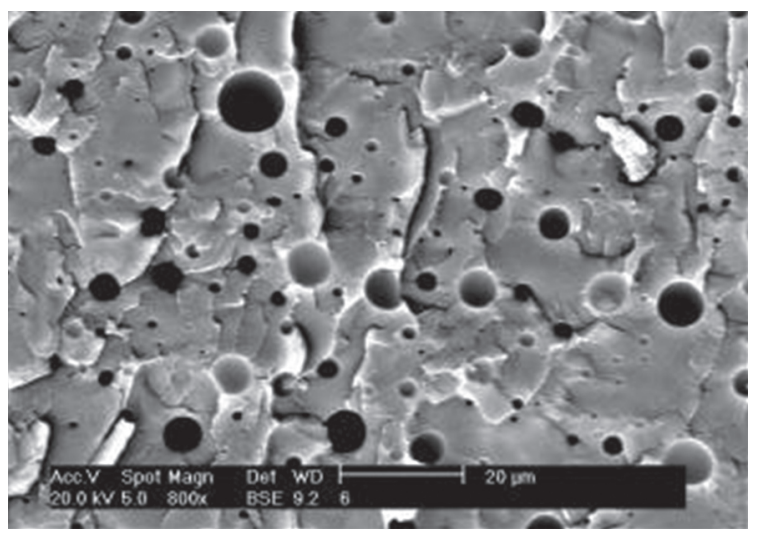

(b)

Figure 4. Micrographs of the material produced using a) 135 minutos $(200 \times)$; and b) 150 minutes $(800 \times)$ of water dispersion time $\left(90^{\circ} \mathrm{C}, 800 \mathrm{rpm}\right.$, DSS, three-bladed propeller stirrer).

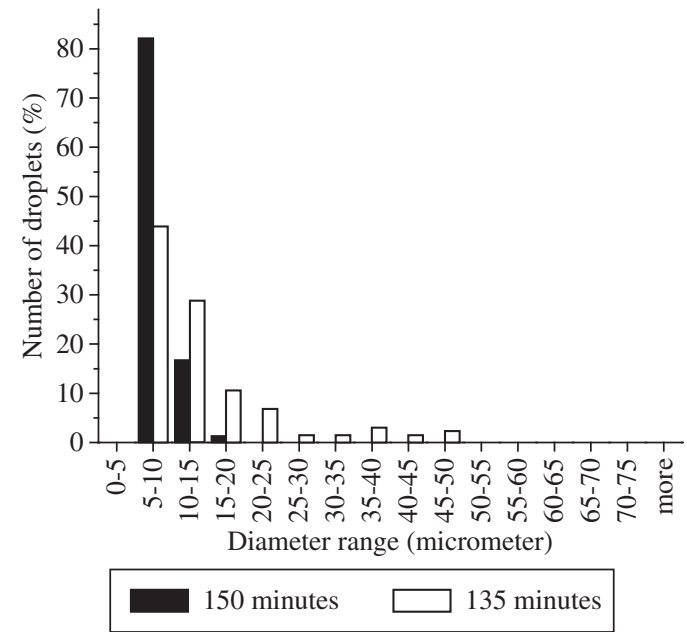

Figure 5. Droplets size distribution obtained using water dispersion times of 135 and 150 minutes $\left(90{ }^{\circ} \mathrm{C}, 800 \mathrm{rpm}\right.$, DSS, three-bladed propeller stirrer).

\section{Influence of the surfactant type}

The Scanning Electron Microscopy images of the internal morphology of the particles obtained using three different surfactants are presented in Figure 6 (images were taken at different magnifications due to the difference in the droplets diameter). The surfactants tested were dodecyl benzene

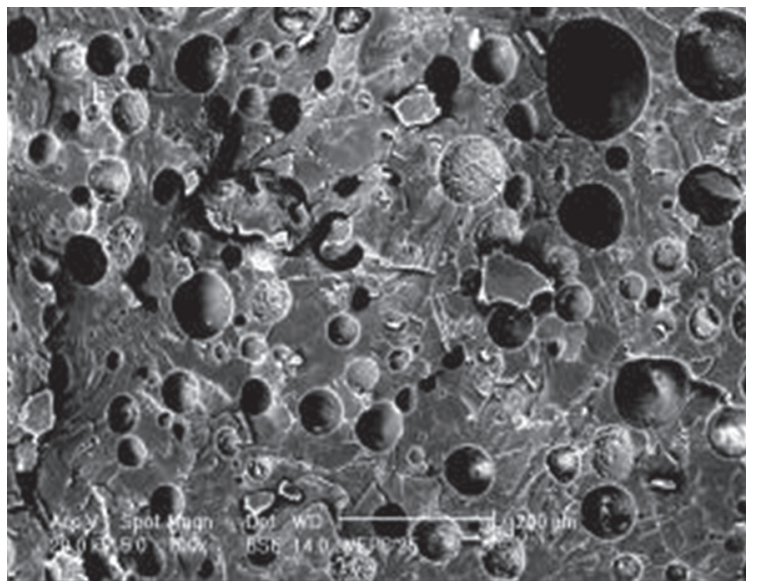

(a)

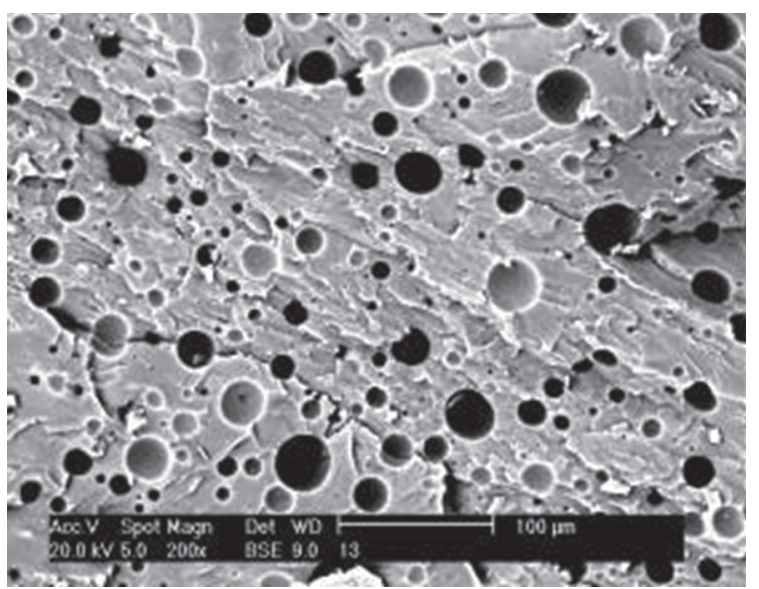

(b)

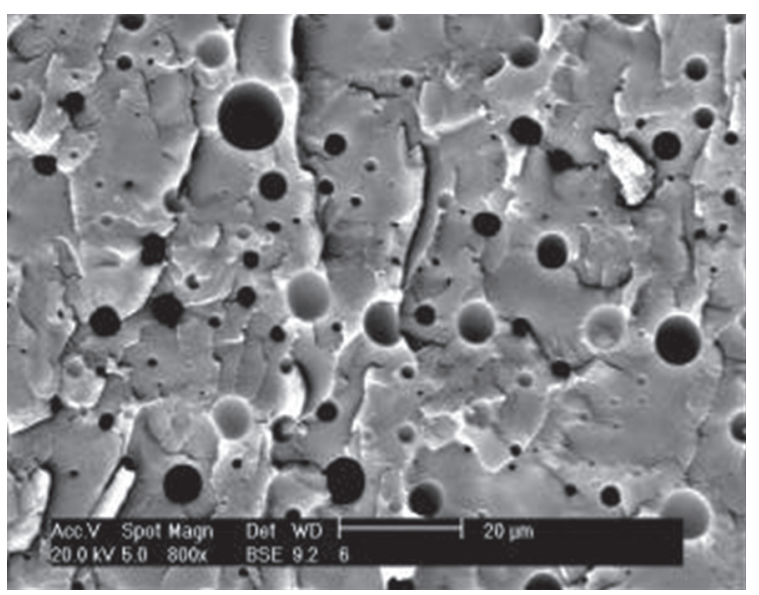

(c)

Figure 6. SEM images of the internal microstructure of the particles obtained using different surfactants. a) DSS (100x); b) DBSS (200x); and c) $\operatorname{AOT}(800 x)$. 


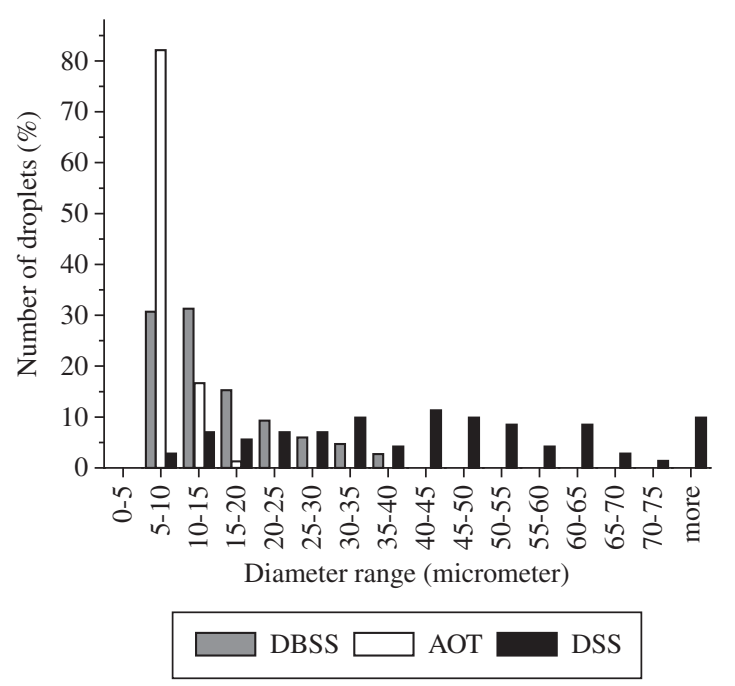

Figure 7. Droplets size distribution obtained using DSS, DBSS or AOT as surfactants $\left(90^{\circ} \mathrm{C}, 800 \mathrm{rpm}\right.$, propeller stirrer, dispersion time of 150 minutes).

sodium sulfonate (DBSS), dodecyl sodium sulfate (DSS) and sodium bis(2-ethylhexyl) sulfosuccinate (AOT). The respective droplets size distributions are showed in Figure 7.

It is possible to observe that the water droplets were uniformly distributed throughout the polymeric matrix for the three surfactants used, since the particles observed were about 1,000 micrometers diameter. AOT favored the formation of more uniform and smaller water droplets than the other surfactants mainly due to the fact that it presents a low Hydrophile-Liphophile Balance - $\mathrm{HLB}^{[7]}$ which favors the stabilization of water-in-oil (w/o) emulsions. DBSS and DSS present high HLB values meaning that they are less effective in stabilizing w/o emulsions.

Comparing the surfactants DBSS and DSS it is possible to observe that DBSS was more efficient in the stabilization of the water droplets inside the monomer-polymer phase. This can be explained by the fact that DBSS presents a larger lipophilic tail than DSS which favors the stabilization of water-in-oil systems ${ }^{[5]}$ because in such systems lipophilic part of the molecule stays in contact with the continuous phase, acting as a steric barrier against coalescence.

\section{Concluding Remarks}

The influence of experimental parameters on the synthesis and internal morphology of water expandable polystyrene particles was investigated. In all cases, water-blowing agent was incorporated in the polystyrene matrix as micro-size droplets distributed homogeneously in the particle. The type of impeller used, the type of surfactant and the duration of the water dispersion phase showed minor influence on the amount of incorporated water. On the other hand, these variables presented a strong effect on the droplets sizes.

AOT was more effective than DBSS and DSS in the stabilization of the water droplets inside the monomer-polymer phase because of its low HLB value. On the other hand, a less stable water-in-oil system was formed when DSS was used because it presents a smaller lipophilic tail when compared to DBSS. The use of a turbine impeller during the water dispersion phase produced smaller droplets than when a three-bladed propeller stirrer was used since the turbine impeller is prone to generate higher shear forces due to the perpendicular flux through the impeller blades. A small increase in the duration of the water dispersion phase led to a decrease in the average water droplets diameter. This can be explained by the fact that the increase in the water dispersion time increased the viscosity of the monomer-polymer phase leading to less coalescence of the water droplets during the suspension polymerization stage.

\section{References}

1. Wittenberg, D. - "Expandable Styrene Polymers", US5096931 (1992).

2. Crevecoeur, J. J.; Nelissen, L. \& Lemstra, P.J. - Polymer, 40, p.3685 (1999a).

3. Crevecoeur, J. J.; Nelissen, L. \& Lemstra, P.J. - Polymer, 40, p.3697 (1999b).

4. Berghmans, H. A. A. - "Process for the preparation of polymer particles", US6127439 (2000).

5. Leal-Calderon, F. \& Poulin, P. - Curr. Opin. Colloid Interface Sci., 4, p.223 (1999).

6. Nogueira, A. L.; Machado, R. A. F. \& Quadri, M. B. Macromol. Symp., 245, p.398 (2006).

7. Tadros, T. F. - "Surfactants", Academic Press, London (1984).

Enviado: 23/04/09

Reenviado: $10 / 08 / 09$

Aceito: 17/08/09 